Review Article

\title{
Indications of sole lift and foot orthoses in the management of mild idiopathic scoliosis -a review
}

\author{
Diego Rothschild, DC ${ }^{1}{ }^{*}$, Shu Yan NG, DC, MS, CPed ${ }^{1)}$, \\ Yin Ling Elaine NG, BS, MSc, MBA, CPed ${ }^{2}$ \\ 1) Hong-Kong Chiropractic College Foundation: 11\&12/F China Hong Kong Tower, \\ 8-12 Hennessy Road, Wan chai, Hong-Kong SAR \\ 2) Wanchai Pedorthic Centre, Hong-Kong SAR
}

\begin{abstract}
Purpose] Leg length discrepancy is common among patients with scoliosis. Some studies reported reduced functional scoliosis curves with correction of leg length discrepancy. Others, however, have shown that induced leg length discrepancy has little effects on spinal deformities. Also, small number of studies assessed the use of foot orthoses in patients with faulty foot biomechanics and their impact on idiopathic scoliosis. In this context, a review of the literature is needed to determine the current evidence for the appropriate use of sole lift and foot orthoses in a context of scoliosis. [Methods] A literature review was performed. [Results] It appeared that sole lifts are indicated for functional lumbar scoliosis when the level of the sacrum is parallel to that of the hips. Sole lifts may not be indicated for patients with structural scoliosis, seemingly inducing a compensatory curve. Custom foot orthoses were found to reduce spinal curves in juvenile patients with mild idiopathic scoliosis and concomitant abnormal foot biomechanics. [Conclusion] Sole lift appeared to be indicated in the presence of certain types of functional scoliosis. Custom foot orthoses can be considered in the management of mild idiopathic scoliosis in juvenile patients. Evidence, however, is low and quality studies are needed to validate these findings. Key words: Adolescent idiopathic scoliosis, Leg length discrepancy, Custom foot orthoses
\end{abstract}

(This article was submitted Aug. 2, 2019, and was accepted Dec. 24, 2019)

\section{INTRODUCTION}

Leg length discrepancy (LLD) is a common condition. The threshold of LLD which is of clinical significance varies with studies and there is at present no universal consensus. LLD of $0.5 \mathrm{~cm}$ is thought to affect approximately half of the population and is associated with an increased prevalence of low back pain ${ }^{1)}$. Epidemiological studies have shown that LLD of less than $2 \mathrm{~cm}$, and more than $0 \mathrm{~cm}$ by definition, affects $40-70 \%$ of the adult population ${ }^{2,3)}$. Moreover, several studies have shown that adolescent idiopathic scoliosis (AIS) is associated with a high prevalence of lower extremities biomechanics abnormalities $^{4-6)}$, including pelvic unlevelness and LLD.

In clinical practice it is not uncommon to see patients with scoliosis and with leg length discrepancy, when it has to be decided whether sole lift or foot orthoses are indicated in the management.

Many studies have attempted to elucidate the relationship between LLDs and scoliosis, but results have not been conclusive. Some studies have shown that correction of LLDs levels pelvic imbalances and reduces the magnitude of functional scoliosis angles ${ }^{1,7-10)}$. Other studies, however, have shown that artificially induced LLDs have little effects on the lumbar curve deformity ${ }^{11,12)}$.

We attempt to review the literature to determine the indications of sole lift and foot orthoses in the management of idiopathic scoliosis.

*Corresponding author. Diego Rothschild (E-mail: rothschild.dc@gmail.com)

(C)2020 The Society of Physical Therapy Science. Published by IPEC Inc.

(c) (1) $\odot$ This is an open-access article distributed under the terms of the Creative Commons Attribution Non-Commercial No Derivatives cc) 


\section{METHODS}

An electronic search was conducted in PUBMED to identify English language papers that examined the relationship between scoliosis, leg length discrepancy and their management with sole lift and foot orthoses. The databases included were from 1980 to 2019 using the key words: scoliosis and leg length discrepancy as well as scoliosis and foot. The literature in foreign language, other than English was excluded. The titles of the remaining papers were then screened. Papers that were solely related to scoliosis, leg length discrepancy or foot biomechanics were excluded and so were papers on secondary scoliosis and surgery. For the remaining papers, the abstracts were read and the relevancy to the present review determined. Only papers that studied or reviewed the relationship between scoliosis and leg length discrepancy or foot biomechanics were included. Full-text copies of these papers were retrieved, evaluated, and relevant information was included in this review.

\section{RESULTS}

In total, the search on scoliosis and leg length discrepancy produced 137 papers, 32 of which were in foreign language. Screening of the titles showed that 92 papers were not relevant. This left 13 papers for review. The search on scoliosis and foot for the period between 1980 and 2019 produced 356 papers, 40 of which were in foreign language. Three hundred and eight papers were not relevant, leaving 8 papers for review.

The review showed that lumbar scoliosis and leg length discrepancy are related. There are different types of LLD and scoliosis. LLD can be subdivided into two groups, structural LLD, when there is structural shortening of lower extremity in one side, and functional LLD, which is a result of altered biomechanics of the lower extremity including the pelvis ${ }^{13)}$. Likewise, scoliosis can be divided into structural and functional scoliosis, both of which have different biomechanical characteristics. Functional scoliosis does not present with a hump in the forward bending test as opposed to structural scoliosis.

Concerning structural scoliosis and how it relates to pelvis disposition and the leg length:

Sekiya et al. ${ }^{14)}$ used EOS imaging system to evaluate 82 AIS patients, to determine if they had anatomical LLDs. They found that structural LLDs were not common in AIS patients as opposed to functional LLDs. Of the cohort, none had structural LLD in excess of $10 \mathrm{~mm}$, whereas 18 of them had functional LLD in excess of $10 \mathrm{~mm}^{14)}$.

The functional LLDs appeared to be correlated with lumbar Cobb angles but not thoracic Cobb angles ${ }^{14)}$. This suggested that functional LLD is possibly compensatory to lumbar curves. The mechanisms involved, however, have not been investigated.

While isolated thoracic scoliosis curve can be compensated before reaching the lumbosacral junction, thoraco-lumbar (TL) and lumbar scoliosis are generally compensated caudally by a lumbosacral hemi curve.

Schwender et al. ${ }^{15)}$ studied 50 AIS patients with left lumbar curve in excess of $40^{\circ}$, in an attempt to determine if the lumbosacral hemicurve predisposed AIS patients to coronal decompensation. They found that in $90 \%$ of cases, the sacrum, representing a component of the hemicurve, was tilted into the lumbar curve and $63 \%$ of patients had an iliac tilt as well. For patients with primary lumbar or TL curves, $100 \%$ had sacral and iliac tilt into the lumbar curve ${ }^{15}$. Sacral tilt could thus be a compensatory mechanism for lumbar curves, which through its connection to the pelvis would contribute to LLD ${ }^{15}$.

Cho et al. ${ }^{16)}$ studied the correlation between sacral slant, pelvic obliquity and lumbar curve angles on radiographs of 303 AIS patients. Their analysis supported the previous findings that pelvic obliquity is linked to sacral tilt, compensating for large lumbar curves ${ }^{16}$. However, the exact cause and effect relationship between the different parameters could not be determined. Also, it is to be noted that partial correlation analysis in this study concluded that LLD was not directly linked with sacral tilt ${ }^{16)}$.

Concerning structural LLD and how it relates to the pelvis disposition and the spinal curvatures:

Multiple studies have attempted to understand the effects of structural LLDs on the pelvic disposition using different tools including motion analysis system, rasterstereography and x-rays. These have shown that LLD affects the pelvis in different planes, including the coronal, sagittal and transverse planes.

Several studies found that LLD is correlated with pelvic tilt in the coronal plane, also called pelvic obliquity 1,2, 13, 14, 16-18).

Some have studied the immediate effects of artificially induced LLDs, using different measuring techniques and showed that LLD affected the pelvic disposition creating pelvic obliquity. Different sizes of sole lifts or elevating platforms were used to induce LLD in healthy participants, including adolescents in one study ${ }^{13)}$ and a group of healthy soldiers in another ${ }^{1)}$ to determine their impact on the pelvic disposition. Results were all consistent, increase in sole height elevating ipsilateral pelvis resulted in pelvic obliquity as illustrated in Fig. 1.

Studies have also shown that LLD affects sagittal pelvic tilt. Betsch et al. ${ }^{11)}$ artificially induced LLDs, varying from 5 to $15 \mathrm{~mm}$, in 115 healthy participants. They found that sole lift increased the sagittal pelvic tilt. It was noted that only half of the increase in sole height was passed onto the sagittal pelvic tilt ${ }^{11)}$. With unclear mechanism, they however thought that this was possibly due to the mobility of the sacroiliac joints, the bony asymmetry of the pelvis and the hypertonic supra-pelvic muscles compensating for some of the sole height difference ${ }^{11)}$.

Many studies have shown that the two innominate bones do not move in tandem in the compensatory pelvic tilt. Beaudoin et al. ${ }^{19)}$ used motion analysis system in 20 female participants to quantify the $3 \mathrm{D}$ postural changes to the pelvis, trunk, 


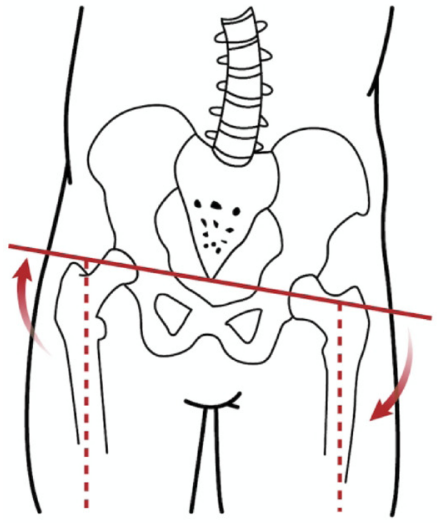

a

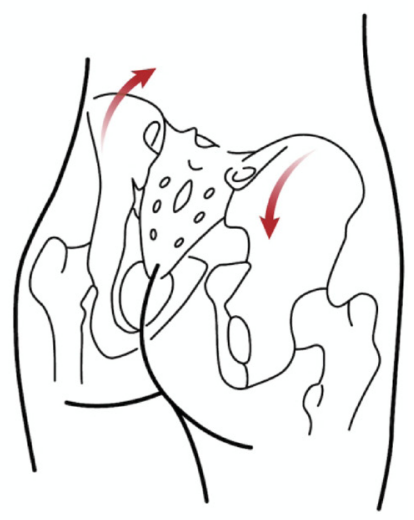

b

Fig. 1. Artificial elevation of sole lift on the right would raise the ipsilateral pelvis and induce a functional left lumbar scoliosis (a). In the sagittal plane, the right sole lift would cause posterior iliac rotation ipsilaterally and anterior iliac rotation contralaterally in the majority of cases (b).

scapular belt and head induced by shoe lift. They showed asymmetric version of the right and left iliac bones in the presence of sole lift, with a posterior rotation of the innominate bone on the side of the sole lift, or long leg, and an anterior rotation on the side of the short leg ${ }^{19)}$ (Fig. 1). These results confirmed the findings by other studies ${ }^{2,20-22)}$. The average total range of version angle was estimated to be 11 degrees ${ }^{22}$. It is of note that some studies ${ }^{23}$ 24), however, showed contradicting results. The iliac bone tended to rotate anteriorly on the side of long leg, and posteriorly on the side of short leg ${ }^{23,24)}$.

Few studies have intended to show the effects of LLD on pelvic disposition in the transverse plane, or pelvic rotation ${ }^{13}$, 19).

Grivas et al. ${ }^{13)}$ using Formetric 4D, found that artificially induced LLD was significantly correlated to pelvic rotation, however the compensatory mechanism was not described.

Concerning structural scoliosis and how it relates to lower extremity biomechanics:

Apart from apparent leg length discrepancy, AIS has been found to be associated with several lower extremity biomechanics abnormalities ${ }^{4-6)}$ including pes valgus ${ }^{4}$, pes cavus ${ }^{25}$, internal leg rotation ${ }^{4}$, increased femoral shaft angle ${ }^{26)}$, increase femoral anteversion/tibial torsion ratio $^{5)}$ and contractures of hip abductors ${ }^{27)}$.

Concerning the prevalence, Lehnert-Schroth ${ }^{4}$, reported a higher incidence of pes valgus in patients with double major scoliosis, thoracolumbar and lumbar curves ${ }^{4}$. Of 115 patients with right thoracic and left lumbar curve, $70.3 \%$ of them presented with pes valgus on the side of thoracic concavity and $20.8 \%$ of them bilaterally. On the side of heel valgus, $79.1 \%$ of the patients had concomitant internal rotation of the leg. On the contrary, $5.2 \%$ of the patients had internal rotation of the leg on the convex side ${ }^{4}$.

Carpintero et $\mathrm{al}^{25}$ ) on the other hand, showed that majority of AIS patients had pes cavus with a prevalence of $65.38 \%$ of the 138 AIS patients examined. Interestingly, the incidence of the pes cavus did not relate to the severity and location of the scoliotic curves ${ }^{25)}$.

Concerning the use of sole lift and foot orthoses in a context of LLD and scoliosis:

In the presence of sacral unlevelness and leg length discrepancy, it is intuitive to add a sole lift on the side of the short leg to level the sacrum and thus reduce the lumbar curve.

Papaionnou et al. ${ }^{7}$ showed that LLD induced scoliosis is non-structural and non-progressive, so called functional scoliosis. Leveling the pelvis by correcting the LLD reduced the lumbar curve. Similarly, Sheha et al. ${ }^{9)}$ and Friberg ${ }^{1)}$ showed that correction of the LLD reduced the pelvic tilt and the functional lumbar scoliosis in a young patient with $20 \mathrm{~mm}$ leg length inequality and in a group of soldiers respectively.

Similar results were seen in a study on 369 adolescents aged 5-17 with functional scoliosis ${ }^{10)}$. Raczkowski et al. ${ }^{10)}$ found that correction of LLD helped reduce the magnitude of scoliosis curves in 316 participants (83.7\%). Interestingly, 80\% of the participants had legs equalization between $8-16$ months of treatment; the mean time required to equalize $1 \mathrm{~cm}$ LLD was about 11.3 months $^{10)}$.

Juhl et al. ${ }^{28)}$ described 6 types of pelvic asymmetries, where the sacral tilt can be parallel to the hip height difference or opposite to the hip height difference, as shown in Fig. 2, seemingly affecting the outcome when using sole lift in the presence of functional lumbar scoliosis.

It is to note that other studies on artificially induced LLD, however, have shown that sole lift ( $5-15 \mathrm{~mm})$ did not change or only changed the spinal postures slightly ${ }^{11,12)}$.

In the presence of structural scoliosis, Lehnert-Schroth ${ }^{4)}$ advised against the use of sole lift as it can induce a compensatory lumbar curve or lumbosacral hemicurve. In the presence of thoracic scoliosis, addition of a sole lift on the side of apparent 
short leg would induce a compensatory lumbar curve. In the presence of lumbar curvature, addition of a sole lift on the side of apparent short leg would induce or enlarge the compensatory lumbosacral hemi-curve ${ }^{4)}$.

Another study has shown that majority of the LLD in structural scoliosis is apparent, and not anatomical ${ }^{14)}$, thus questioning the use of sole lift in the presence of structural scoliosis.

Apart from sole lift, underfoot wedges and custommade foot orthoses have also been found to influence spinal curves in AIS patients ${ }^{29}$.

D'Amico et al. ${ }^{29)}$ used the opto-electronic approach to evaluate the effects of wedges of different sizes on posture of 220 patients aged between 5-19 years of age with mild to severe idiopathic scoliosis. They found that application of underfoot wedges resulted in significant postural improvements in re-balancing and in spinal deformities in 193 patients $(87.7 \%)$; the remaining $12.3 \%$ of the patients did not respond to the intervention ${ }^{29)}$.

Recently, Park et al. ${ }^{30)}$ and Lee et al. ${ }^{31)}$ reported an improvement in Cobb angle in scoliosis patients treated with custom made foot orthoses. Park et al. ${ }^{30)}$ used Formetric $4 \mathrm{D}$ to evaluate the effects of biomechanical foot orthoses on 26 scoliosis patients, aged between 13-60 years of age. They found that the foot orthoses significantly reduced the pelvic obliquity and the scoliosis angle.

Lee et al. ${ }^{31)}$ did a similar study, but with a more homogeneous group of patients. They prescribed custom foot orthoses to 52 juvenile idiopathic scoliosis patients with a mean age of 79.5 months, with foot pronation ${ }^{31)}$. They found that weight bearing heel valgus angle (the resting calcaneal stance phase angle) reduced from a mean of $4.25^{\circ}$ to $1.71^{\circ}$ and the pelvic height difference reduced from a mean of $1.07 \mathrm{~cm}$ to $0.60 \mathrm{~cm}^{31}$ ) More importantly perhaps, patients less than 6 years of age had a significant improvement of Cobb angle of more than $5^{\circ 31)}$. The Cobb angle improved most at 9 months of the wear ${ }^{31)}$ Improvement of Cobb angle, however, was not seen in patients with curve in excess of $25^{\circ}$.

\section{DISCUSSION}

From the studies, it is apparent that prescription of sole lift and custom foot orthoses depends on a number of factors. Sole lift seems to be indicated in the presence of functional lumbar scoliosis on the side of short leg ${ }^{1,7-10)}$, and in line with Juhl et al. ${ }^{28)}$ findings we believe that it should only be applied when the level of the sacrum is parallel to that of the hips ${ }^{28)}$ (Fig. 2). In the presence of idiopathic scoliosis, it is essential to check if anatomical short leg does exist, as functional LLD is more frequent ${ }^{14}$. Addition of sole lift in the absence of anatomical LLD may induce a compensatory lumbar or lumbosacral curve ${ }^{4)}$.

The prescription of custom foot orthosis, however, is less certain. Despite the recent findings that custom foot orthoses would reduce the spinal curves in juvenile patients with idiopathic scoliosis ${ }^{30}$ ) and in patients with scoliosis ${ }^{31)}$, the SOSORT 2011 guidelines stipulated that there was little or no evidence to support the use of custom foot orthosis in the management of idiopathic scoliosis ${ }^{32)}$.

The authors think that it is possible that the clinical effectiveness of custom foot orthoses depends on the magnitude and flexibility of the curve and whether the patient has abnormal foot biomechanics. In juvenile patients with scoliosis less than $25^{\circ}$ and asymmetric abnormal foot biomechanics, custom foot orthoses have been found to reduce the Cobb angle over 9 months ${ }^{31}$.

We believe that in young patients with mild idiopathic scoliosis and sufficient spinal flexibility, the correction of the asymmetric abnormal foot biomechanics induces corrective movements in the lower extremities that are transmitted to the pelvis and the spine and reducing the angle of scoliosis (Fig. 3). On the other hand, we think that with increase in curve and reduced spinal flexibility, the correction of the asymmetric abnormal foot biomechanics may not reduce the Cobb angle, as some of the transverse plane corrective movements induced by the custom foot orthoses may be taken up by the knees, hips and sacroiliac joints, instead of being transmitted to the spine. Moreover, once the curve is moderate in magnitude and is structural, the force induced by the foot orthoses would not be sufficient to cause a decrease in spinal curve. This is akin to the treatment of tibial varum in children under 2 years of age ${ }^{33)}$. Abstinence from weight bearing in the first two years of life, together with vitamin D3 supplementation were reported to reduce tibial varum ${ }^{33)}$, but they are certainly of little use in the treatment of medial compartment knee arthrosis, which is the end stage of tibial varum. Similarly, we opined that custom 


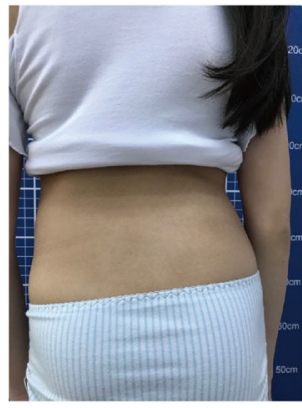

a

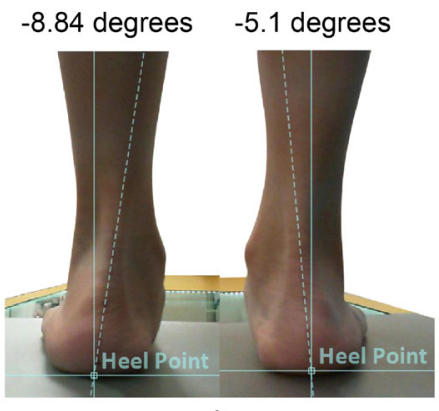

b

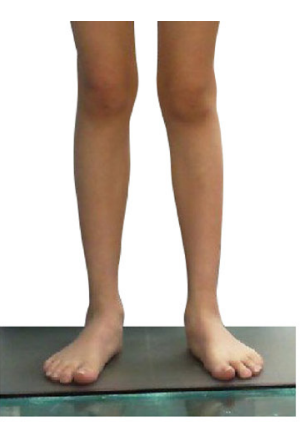

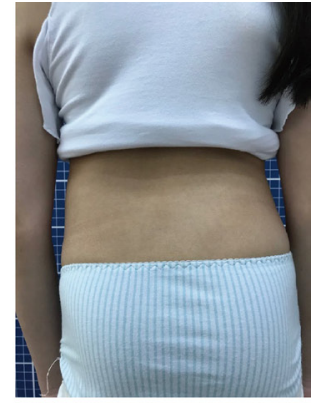

d

Fig. 3. Custom foot orthoses for management of abnormal foot biomechanics. (a) It can be seen that the left posterior superior iliac spine is lower than that of the right. (b) Physical examination of the patient revealed bilateral forefoot varus. The relaxed calcaneal stance phase of the left heel is greater than that of the right. (c) The feet of the patient when seen from the front. (d) When wearing the prescription custom foot orthoses (without heel or sole lift) to correct the abnormal foot biomechanics, the posterior superior iliac spines on both sides become level.

foot orthoses might be indicated in the early stage of idiopathic scoliosis but not when the scoliosis increases beyond $25^{\circ}$.

The available evidence suggested that sole lifts are recommended in the presence of functional scoliosis, when the level of the sacrum is parallel to that of the hips and custom foot orthoses may be indicated in the management of early stage of mild idiopathic scoliosis, particularly to juvenile patients with abnormal foot biomechanics. However, the available evidence is of low quality and further studies are required to validate their effectiveness.

\section{Funding and Conflict of interest}

The authors declare that they have no funding nor conflict of interest.

\section{REFERENCES}

1) Friberg O: Clinical symptoms and biomechanics of lumbar spine and hip joint in leg length inequality. Spine, 1983, 8: 643-651. [Medline] [CrossRef]

2) Cummings G, Scholz JP, Barnes K: The effect of imposed leg length difference on pelvic bone symmetry. Spine, 1993, 18: 368-373. [Medline] [CrossRef]

3) Guichet JM, Spivak JM, Trouilloud P, et al.: Lower limb-length discrepancy. An epidemiologic study. Clin Orthop Relat Res, 1991, (272): 235-241. [Medline]

4) Lehnert-Schroth C: Three-dimensional treatment for scoliosis. California: The Martindale Press, 2007.

5) Burwell RG, Aujla RK, Kirby AS, et al.: Ultrasound femoral anteversion (FAV) relative to tibial torsion (TT) is abnormal after school screening for adolescent idiopathic scoliosis (AIS): evaluation by two methods. Stud Health Technol Inform, 2008, 140: 37-43. [Medline]

6) Białek M: Mild angle early onset idiopathic scoliosis children avoid progression under FITS method (Functional Individual Therapy of Scoliosis). Medicine (Baltimore), 2015, 94: e863. [Medline] [CrossRef]

7) Papaioannou T, Stokes I, Kenwright J: Scoliosis associated with limb-length inequality. J Bone Joint Surg Am, 1982, 64: 59-62. [Medline] [CrossRef]

8) Irvin RE: Reduction of lumbar scoliosis by use of a heel lift to level the sacral base. J Am Osteopath Assoc, 1991, 91: 34, 37-44. [Medline]

9) Sheha ED, Steinhaus ME, Kim HJ, et al.: Leg-length discrepancy, functional scoliosis, and low back pain. JBJS Rev, 2018, 6: e6. [Medline] [CrossRef]

10) Raczkowski JW, Daniszewska B, Zolynski K: Functional scoliosis caused by leg length discrepancy. Arch Med Sci, 2010, 6: 393-398. [Medline] [CrossRef]

11) Betsch M, Wild M, Große B, et al.: The effect of simulating leg length inequality on spinal posture and pelvic position: a dynamic rasterstereographic analysis. Eur Spine J, 2012, 21: 691-697. [Medline] [CrossRef]

12) Kwon YJ, Song M, Baek IH, et al.: The effect of simulating a leg-length discrepancy on pelvic position and spinal posture. J Phys Ther Sci, 2015, 27: 689-691. [Medline] [CrossRef]

13) Grivas TB, Angouris K, Chandrinos M, et al.: Truncal changes in children with mild limb length inequality: a surface topography study. Scoliosis Spinal Disord, 2018, 13: 27. [Medline] [CrossRef]

14) Sekiya T, Aota Y, Yamada K, et al.: Evaluation of functional and structural leg length discrepancy in patients with adolescent idiopathic scoliosis using the EOS imaging system: a prospective comparative study. Scoliosis Spinal Disord, 2018, 13: 7. [Medline] [CrossRef]

15) Schwender JD, Denis F: Coronal plane imbalance in adolescent idiopathic scoliosis with left lumbar curves exceeding 40 degrees: the role of the lumbosacral hemicurve. Spine, 2000, 25: 2358-2363. [Medline] [CrossRef]

16) Cho JH, Lee CS, Joo YS, et al.: Association between sacral slanting and adjacent structures in patients with adolescent idiopathic scoliosis. Clin Orthop Surg, 2017, 9: 57-62. [Medline] [CrossRef]

17) Needham R, Chockalingam N, Dunning D, et al.: The effect of leg length discrepancy on pelvis and spine kinematics during gait. Stud Health Technol Inform, 2012, 176: 104-107. [Medline]

18) Coffey D, Hudson-Phillips SP, Radha SS, et al.: Leg length discrepancy: the importance of a complete history and examination. BMJ Case Rep, 2017, 2017: bcr-2017-220985. [Medline] [CrossRef] 
19) Beaudoin L, Zabjek KF, Leroux MA, et al.: Acute systematic and variable postural adaptations induced by an orthopaedic shoe lift in control subjects. Eur Spine J, 1999, 8: 40-45. [Medline] [CrossRef]

20) Kappler RE: Postural balance and motion patterns. J Am Osteopath Assoc, 1982, 81: 598-606. [Medline]

21) Subotnick SI: Limb length discrepancies of the lower extremity (the short leg syndrome). J Orthop Sports Phys Ther, 1981, 3: 11-16. [Medline] [CrossRef]

22) Pitkin HC, Pheasant HC: Sacrarthrogenetic telalgia. J Bone Joint Surg Am, 1936, 18: 365-374.

23) Cibulka T, Koldenhoff R: Leg length disparity and its effect on SI joint dysfunction. Clinical Management in Phys Ther, 1986, 6: 10-11.

24) Thomas PE: An analysis of the interactions among various asymmetric osseous pelvic and lumbar structures. J Am Osteopath Assoc, 1965, 64: 956-957.

25) Carpintero P, Entrenas R, Gonzalez I, et al.: The relationship between pes cavus and idiopathic scoliosis. Spine, 1994, 19: 1260-1263. [Medline] [CrossRef]

26) Saji MJ, Upadhyay SS, Leong JC: Increased femoral neck-shaft angles in adolescent idiopathic scoliosis. Spine, 1995, 20: 303-311. [Medline] [CrossRef]

27) Karski T: New clinical observations connected with "biomechanical aetiology of so called idiopathic scoliosis" (2006-2007). Stud Health Technol Inform, 2008, 140: 194-196. [Medline]

28) Juhl JH, Ippolito Cremin TM, Russell G: Prevalence of frontal plane pelvic postural asymmetry—part 1. J Am Osteopath Assoc, 2004, 104: 411-421. [Medline]

29) D'Amico M: Scoliosis and leg asymmetries: a reliable approach to assess wedge solutions efficacy. Stud Health Technol Inform, 2002, 88: 285-289. [Medline]

30) Park SM, Ahn SH, Lee AY, et al.: Raster-stereographic evaluation of the effects of biomechanical foot orthoses in patients with scoliosis. J Phys Ther Sci, 2016, 28: 1968-1971. [Medline] [CrossRef]

31) Lee JG, Yun YC, Jo WJ, et al.: Correlation of radiographic and patient assessment of spine following correction of nonstructural component in juvenile idiopathic scoliosis. Ann Rehabil Med, 2018, 42: 863-871. [Medline] [CrossRef]

32) Negrini S, Aulisa AG, Aulisa L, et al.: 2011 SOSORT guidelines: Orthopaedic and Rehabilitation treatment of idiopathic scoliosis during growth. Scoliosis, 2012, 7: 3. [Medline] [CrossRef]

33) Karski T. http://www.ortopedia.karski.lublin.pl/p/photo.php?id=3423\&o=d\&u=all. (Accessed May 30, 2019) 